\title{
Operation and Performance of the Mid-infrared Camera, NOMIC, on the Large Binocular Telescope
}

\author{
William F. Hoffmann ${ }^{\mathrm{a}}$, Philip M. Hinz ${ }^{\mathrm{a}}$, Denis Defrère ${ }^{\mathrm{a}}$, Jarron M. Leisenring ${ }^{\mathrm{a}}$, Andrew J Skemer ${ }^{\mathrm{a}}$, \\ Paul A. Arbo ${ }^{\mathrm{a}}$, Manny Montoya ${ }^{\mathrm{a}}$, Bertrand Mennesson ${ }^{\mathrm{b}}$ \\ ${ }^{\text {a }}$ Steward Observatory, The University of Arizona \\ ${ }^{\mathrm{b}}$ Jet Propulsion Lab, California Institute of Technology
}

\begin{abstract}
The mid-infrared ( $8-13 \mu \mathrm{m})$ camera, NOMIC, is a critical component of the Large Binocular Telescope Interferometer search for exozodiacal light around near-by stars. It is optimized for nulling interferometry but has general capability for direct imaging, low resolution spectrometry, and Fizeau interferometry. The camera uses a Raytheon 1024x1024 Si:As IBC Aquarius array with a $30 \mu \mathrm{m}$ pitch which yields 0.018 arc-second pixels on the sky. This provides spatial resolution $(\lambda / \mathrm{D})$ at a $10 \mu \mathrm{m}$ wavelength of 0.27 arc-seconds for a single 8.4 meter LBT aperture and of 0.10 arcseconds for Fizeau interferometry with the dual apertures. The array is operated with a differential preamplifier and a version of the 16 channel array controller developed at Cornell University for the FORCAST instrument on the Sofia Observatory. With a 2.4 MHz pixel rate the camera can achieve integration times as short as 27 milliseconds full array and 3 milliseconds partial array. The large range of integration times and two array integration well sizes allow for a wide range of background flux on the array. We describe the design and operation of the camera and present the performance of this system in terms of linearity, noise, quantum efficiency, image quality, and photometric sensitivity.
\end{abstract}

Keywords: Infrared astronomy, IBC detector, LBT, nulling interferometry, exozodiacal light

\section{THE CONTEXT}

The goal of this work is to provide a ground-based astronomical instrument for mid-infrared $(8-13 \mu \mathrm{m})$ high contrast imaging of nearby stars to enable detection and measurement of exozodiacal light and extra-solar planets. The instrument is being constructed for the Large Binocular Telescope (LBT) operated by the partners, Arizona, Italy, Germany, The Research corporation, and Ohio State University . The telescope (Figure 1-1) is located on Mt Graham, Arizona at an altitude of 3191 meters (10470 feet). Adaptive optics thin shell secondaries provide a Strehl ratio of 0.98 at $11 \mu \mathrm{m}^{1}$.

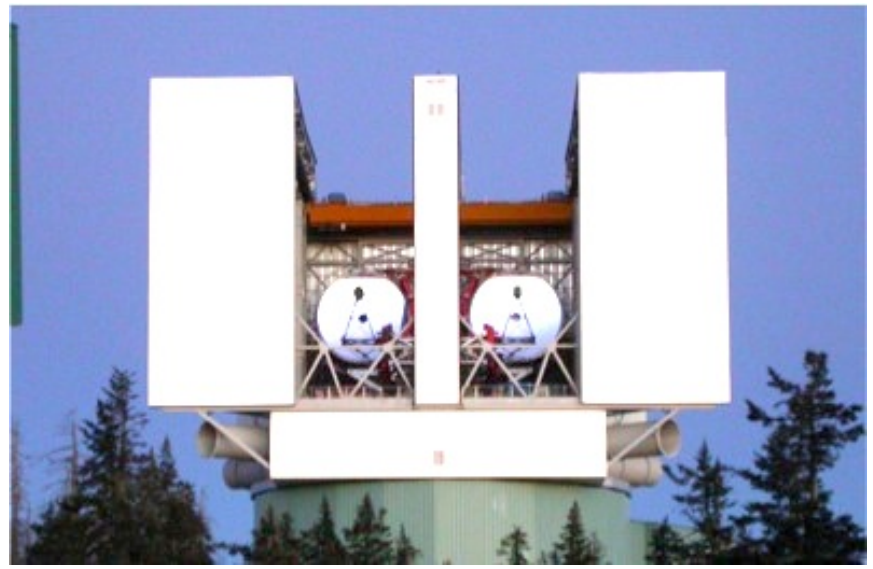

Figure 1-1 Large Binocular Telescope showing the two 8.4 meter primary mirrors with 22.7 meter edge-to-edge spacing. 
The Large Binocular Telescope Interferometer $\left(\mathrm{LBTI}^{2,3,4}\right)$, shown in Figure 1-2, brings the beams from the two telescopes of the LBT to a common focus. The LBTI provides a rigid external structure for a cryogenically cooled beam train and slow alignment mechanisms and fast atmospheric phase tip tilt correction. Figure 1-3 shows the optical path through the beam combiner to the Nulling and Imaging Camera (NIC) ${ }^{5}$ with its three subsystems, LMIRcan $(1-5 \mu \mathrm{m})^{6}$, Phasecam $(2-2.4 \mu \mathrm{m})$, and NOMIC $(8-13 \mu \mathrm{m})$.

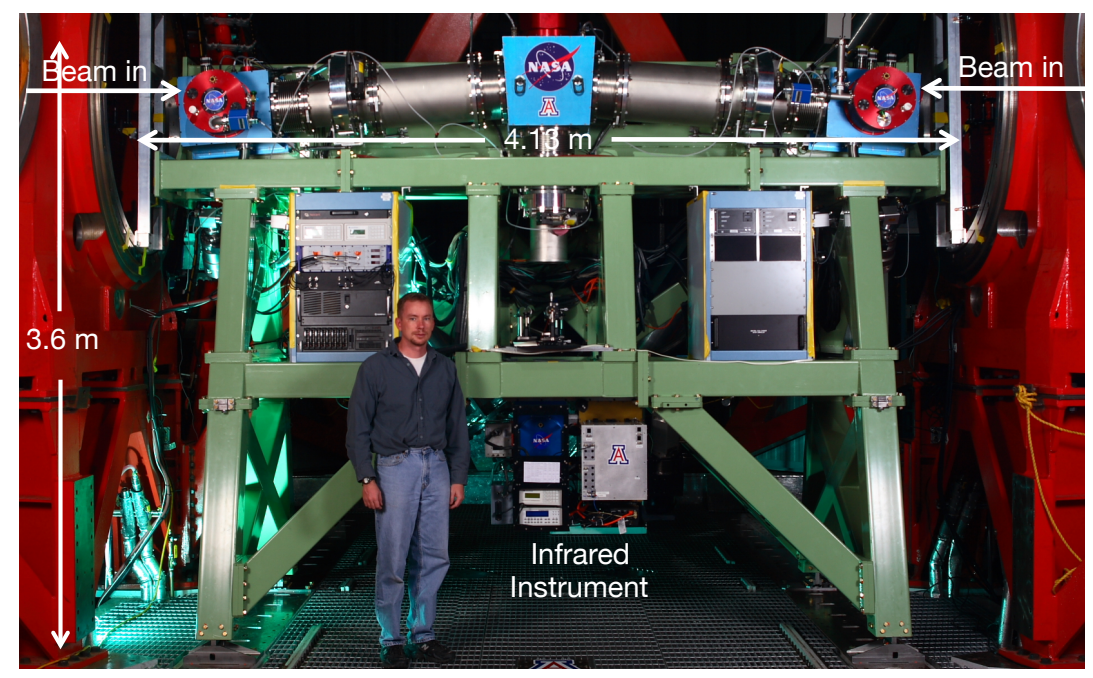

Figure 1-2 The LBT Interferometer with PI, Phil Hinz. This shows the rigid steel support structure, the vacuum enclosed beam combiner with light beams entering from the left and right telescopes, three blue boxes for cryogenically cooled optics, and the infrared instrument mechanically cooled to cryogenic temperatures

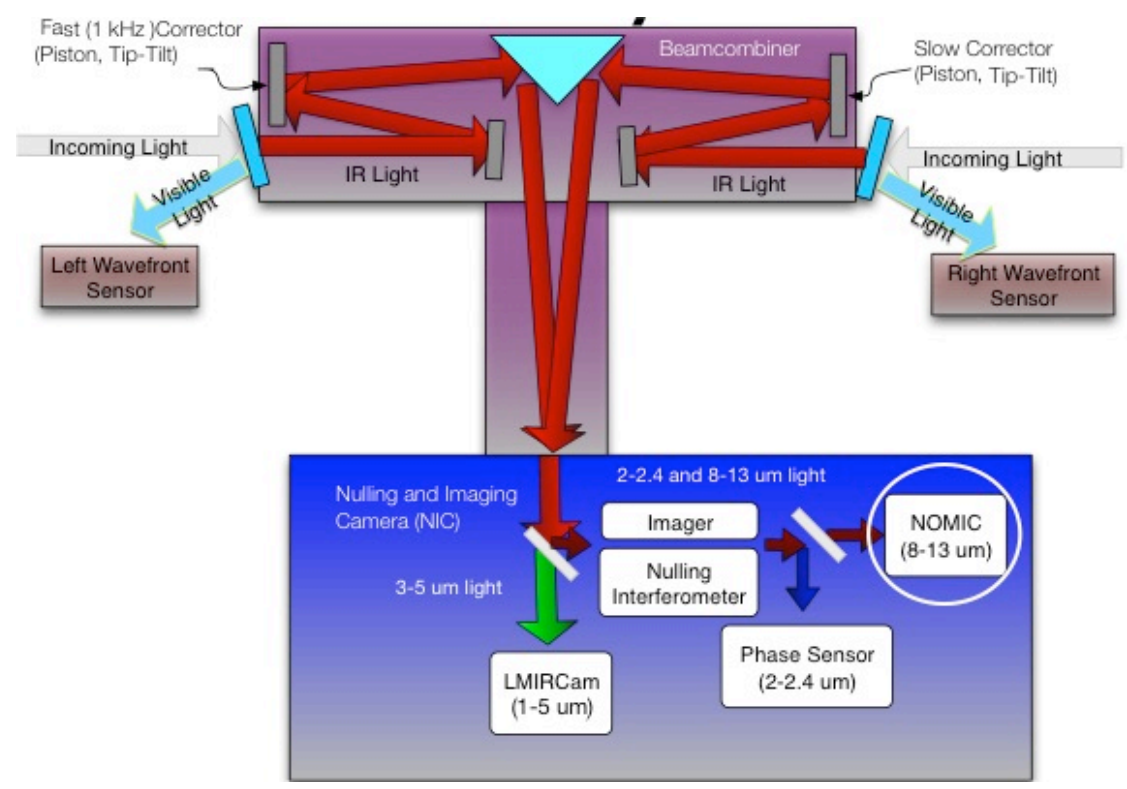

Figure 1-3 Components of the LBT Interferometer showing the optical path through the beam combiner and the camera. The beams combiner includes a fast $(1 \mathrm{kHz})$ piston, tiptilt corrector on the left side and a slow, larger range, corrector on the right. The cooled instrument enclosure includes a 1-5 $\mu \mathrm{m}$ near-infrared astronomical camera, LMIRcam, a 2-2.4 $\mu \mathrm{m}$ phase sensing camera, and the $8-13 \mu \mathrm{m}$ mid-infrared camera, NOMIC. 


\section{THE INSTRUMENT}

Figure 2-1 shows the optical path for NOMIC, the Nulling Optimized Mid-Infrared Camera, from the combined focal plane to the Raytheon Aquarius Detector. The detector is a 1024x1024 Impurity Band Conductor (IBC) hybrid array with $30 \mu \mathrm{m}$ pixels [2]. The optics provides a field of view of 12 arc-seconds and 0.018 arc-second pixels. $\lambda / \mathrm{D}$ for an individual aperture at $11 \mu \mathrm{m}$ is 0.27 arc-seconds or 15 pixels. For Fizeau interferometry with the two apertures it is 0.10 arc-seconds or 5.5 pixels.

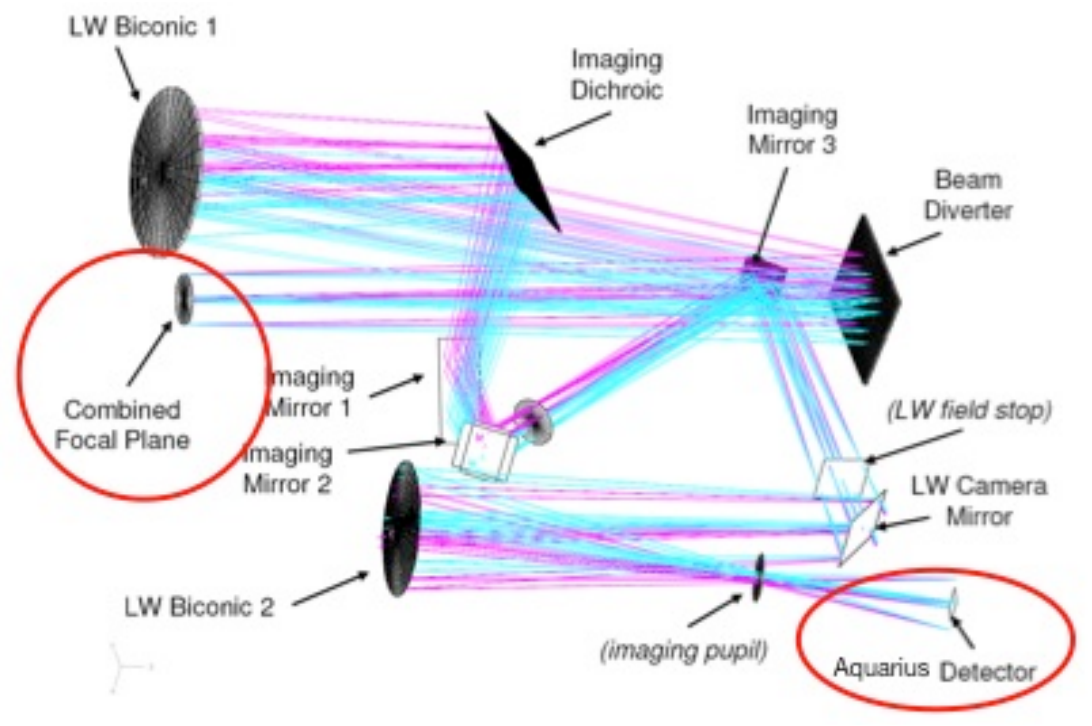

Figure 2.1 Nulling Optimized Infrared Camera (NOMIC) showing folding and reimaging mirrors from the input combined focal plane to the Aquarius detector.

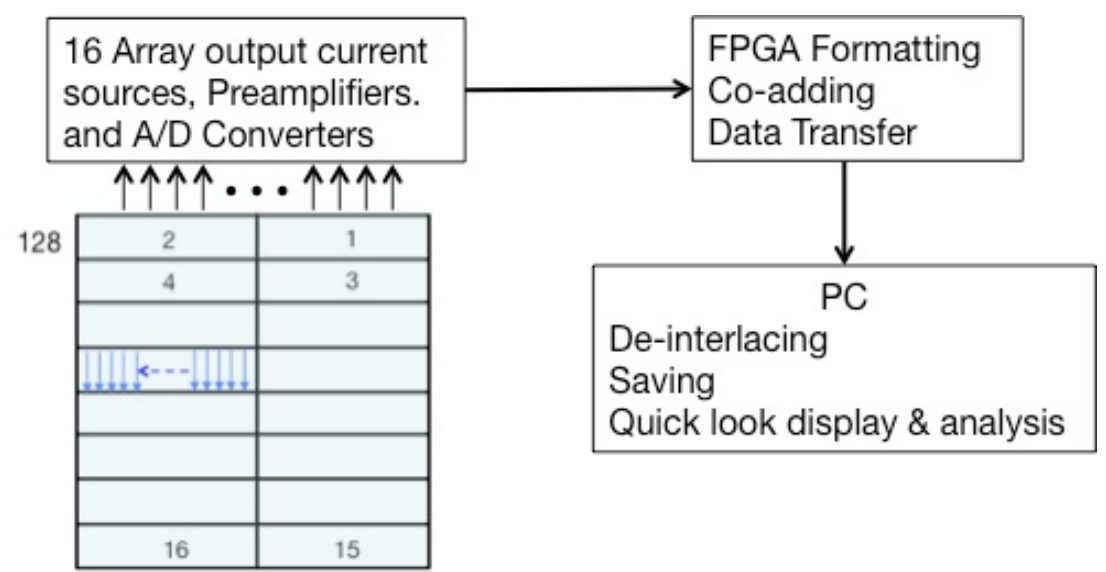

Array channels and readout

Figure 2-2 Array 16 channel control electronics. The vertical arrows show the direction of the sequential readout of channel 8. The full array can be read out in $27 \mathrm{msec}$. At reduced widths such as 512, 256, or 128 pixels, it can be read out in as little as $3 \mathrm{msec}$. 
Figure 2-2 shows a block diagram of the array control electronics, which is the Cornell University FORCAST system ${ }^{7}$ developed for the NASA SOFIA observatory. The array is formatted with 16 channels each reading out a $128 \times 512$ block of pixels. The channels are multiplexed in a "rolling mode" with each column reset as the next column is read. A subarray mode allows for reduced width and greater readout speed, e.g. 512, 256, or 128 pixels. A 14 bit A/D provides for $2.4 \mathrm{MHz}$ pixel read rate. For the full array with 65536 pixels per channel the read time is 27 milliseconds. For a partial array it is $\geq 3$ milliseconds. The array has two selectable integrating capacitors, high and low gain for $10^{6}$ and $10^{7}$ electron well size

The Aquarius array source follower output has a voltage of $6.5+/-0.5$ and requires a high impedance current source whereas our controller signal processor input can handle only $+/-4 \mathrm{~V}$ with a low impedance current source. Therefore we require an interface between the two. Figure 2-3 shows the circuit diagram of one channel of a 16 channel preamplifier which provides the required voltage range and current source.

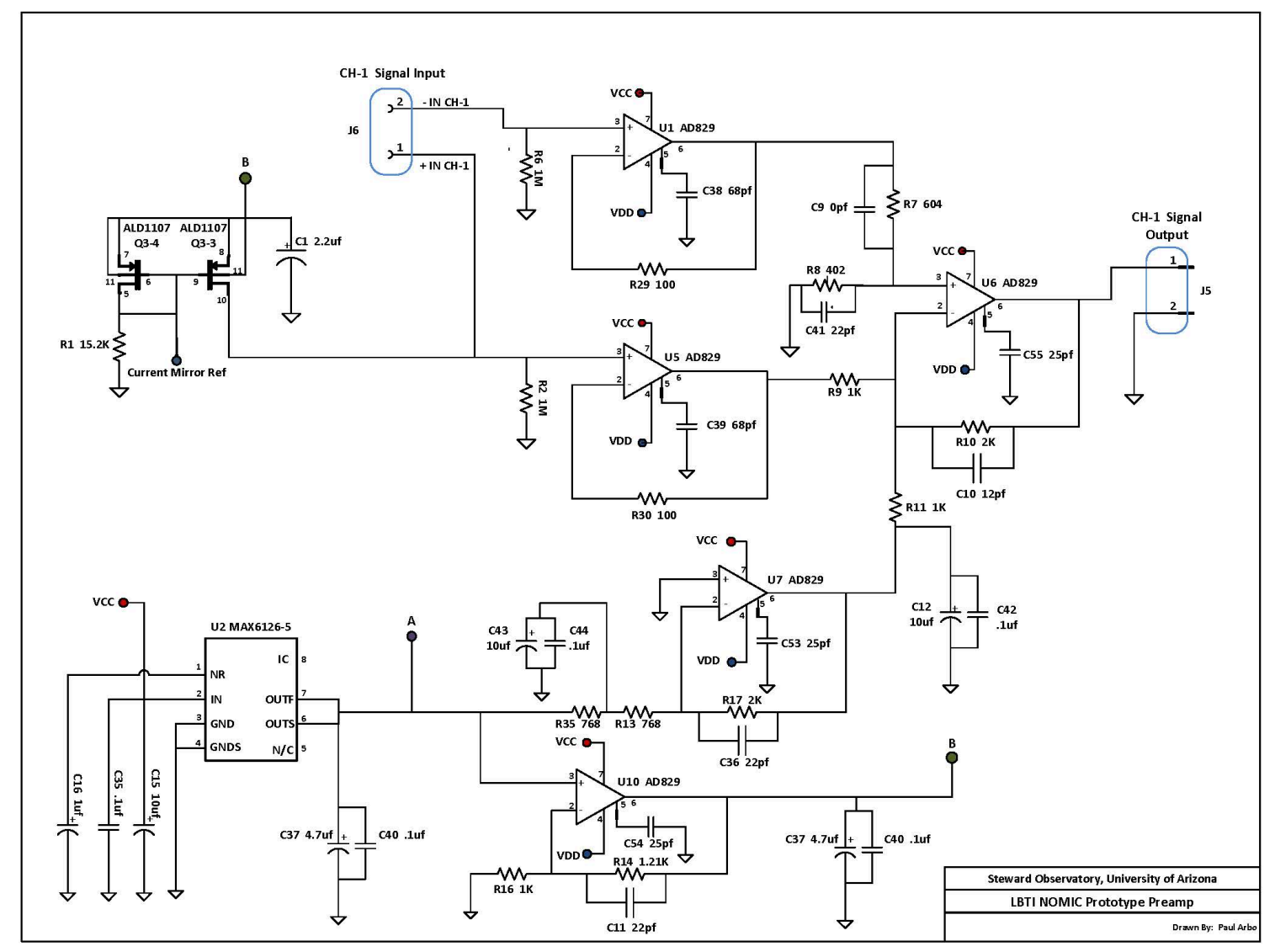

Figure 2-3 Circuit diagram of a single channel of the NOMIC preamplifier. The preamplifier provides a differential input with the reference level at ground (-in) and signal level at $6.5+/-0.5 \mathrm{~V}(+\mathrm{in})$. The + input is supplied with a 0.5 $\mathrm{mA}$ current from a current mirror utilizing an ALD1 107 pchannel MOSFET matched pair. The operational amplifiers for the differential input, single ended output, and $6.5 \mathrm{~V}$ offset are low noise, high-frequency AD829. The preamplifier provides the required $5 \mathrm{MHz}$ bandwidth and adds negligibly the array and controller noise.

\section{PERFORMANCE}

The performance and noise measurements were made with an "engineering" grade array rather than a "science" grade array. All measurements were made with high detector gain. Figure 3-1 is a linearity plot showing good linearity from $12 \%$ to $84 \%$ of saturation. It is likely that the glitch starting at $84 \%$ can be removed by a bias change. 
Figure 3-2 shows the read and shot noise as a function of well filling. At low well filling it is dominated by read noise. At high well filling the shot noise becomes dominant. The read noise is a factor of four above the manufacturer's specification. A quadratic fit to the measured noise with the read noise subtracted is consistent with a quantum efficiency of 0.5. This agrees with the manufacturer's specification. However, as shown in Figure 3-2, the noise does not continue to rise with well filling as is expected from shot noise.

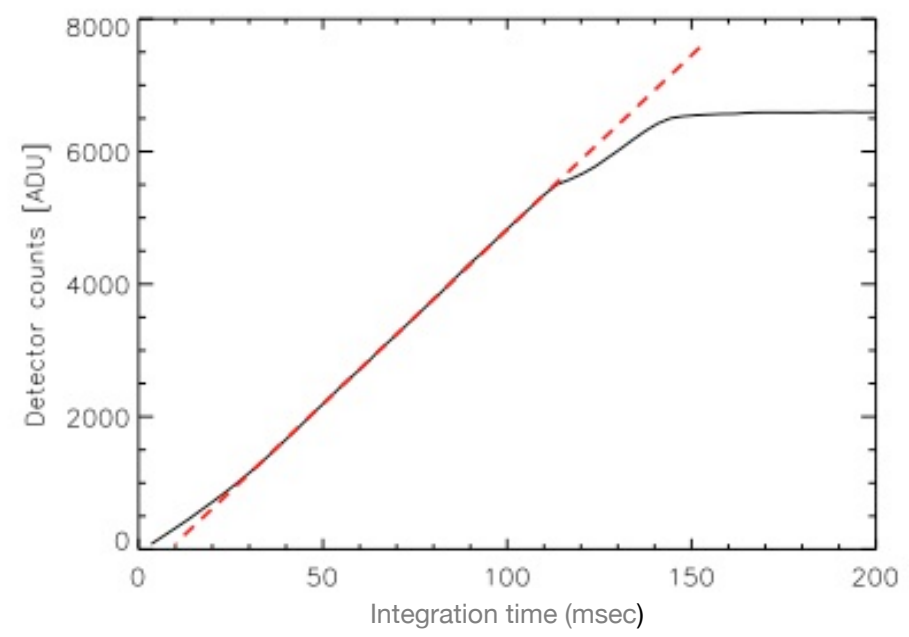

Figure 3-1 Plot of detector flux in digital units versus integration time in milliseconds. This shows good linearity from $12 \%$ to $84 \%$ of saturation. One ADU is 157 electrons.

NOMIC Aquarius Flt-115 Noise versus Flux 13-Jun-2014 ID 972 Flux w/ filters

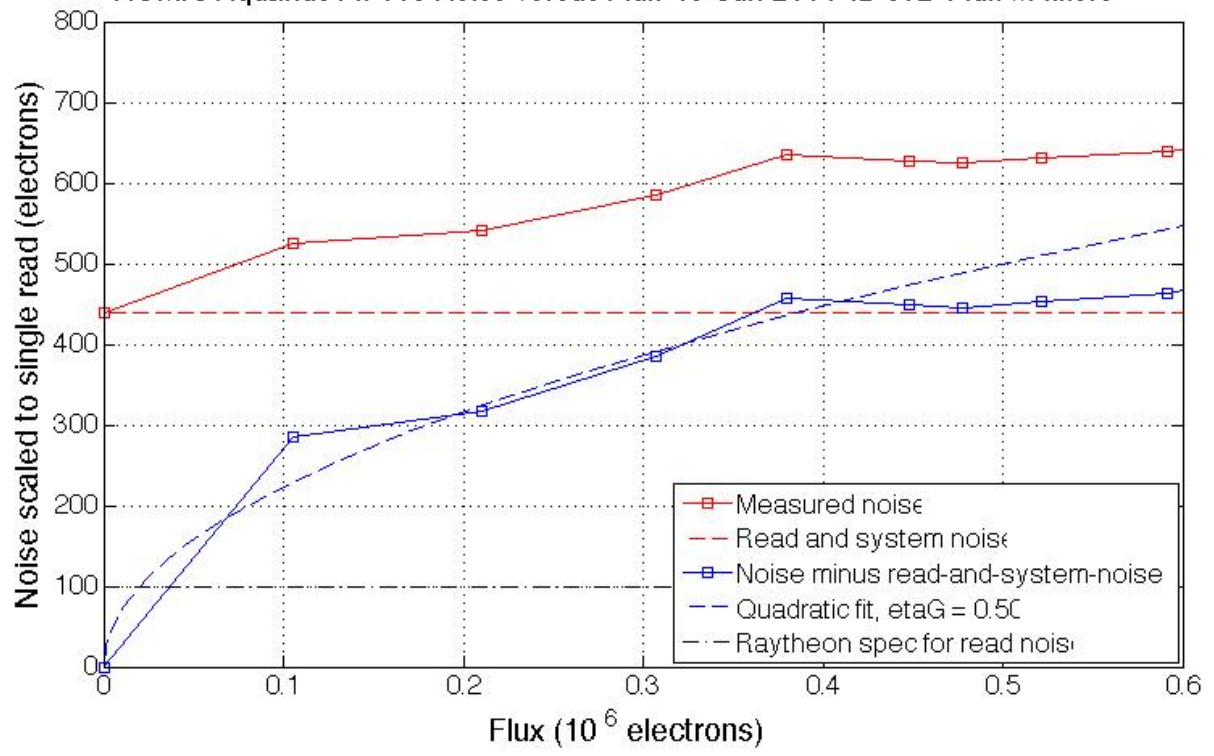

Figure 3-2 Read and shot noise in electrons versus well filling in electrons. The upper solid line is the total measured noise. The upper dashed line is the blanked-off noise (read noise). This is four times the manufacturer's specification shown as the lower dot-dashed line. The lower solid line is the read noise subtracted from the measured noise in quadrature. The dashed line is a quadratic fit calculated for shot noise with $\eta \mathrm{G}$ (quantum efficiency times photoconductive gain) $=0.5$. This difference behaves as shot noise up to about 450 electrons where it stops rising for an unknown reason. The array saturates at $1.0 \times 10^{6}$ electrons. 
From extensive testing we believe that the high noise does not originate in the controller, electronics, wiring, or our operation of the array. It also appears not to arise in the detector itself. In the summer of 2014 we will substitute a bare mux for the array for further diagnostics.

The photometric sensitivity on the sky with the LBT utilizing both apertures has been measured with standard stars. The sensitivity is $0.37 \mathrm{mJy}, 3$ sigma, in 1 hour measured in $\mathrm{N}^{\prime}$ band $(9.8-12.4 \mu \mathrm{m})$. The images were taken rapidly with low detector well filling and with the high read noise dominating. Sky and telescope background subtraction was obtained by "nodding" the telescope on and off the star with a period of approximately 2 minutes. Not included in the 60 minutes is a controller overhead which for this measurement was $30 \%$ and can be smaller or larger depending on the size of the sub-array processed and the controller mode.

Figure 3-3 shows the image quality for a point source and the noise away from the source. Both are satisfactory. There is an artifact which causes a depression in the readout from a bright source which shows faintly below the source. This appears to be due to a depression in the level of each channel as it reads over a bright source. This artifact can be totally removed by a simple post-processing algorithm.

Figure 3-4 shows the performance of the system when used as a Fizeau interferometer. The detector provides 8 pixels per fringe period. The short exposure shown illustrates an interferometric PSF that is, to the limits of the signal-to-noise, indistinguishable from a theoretical PSF.
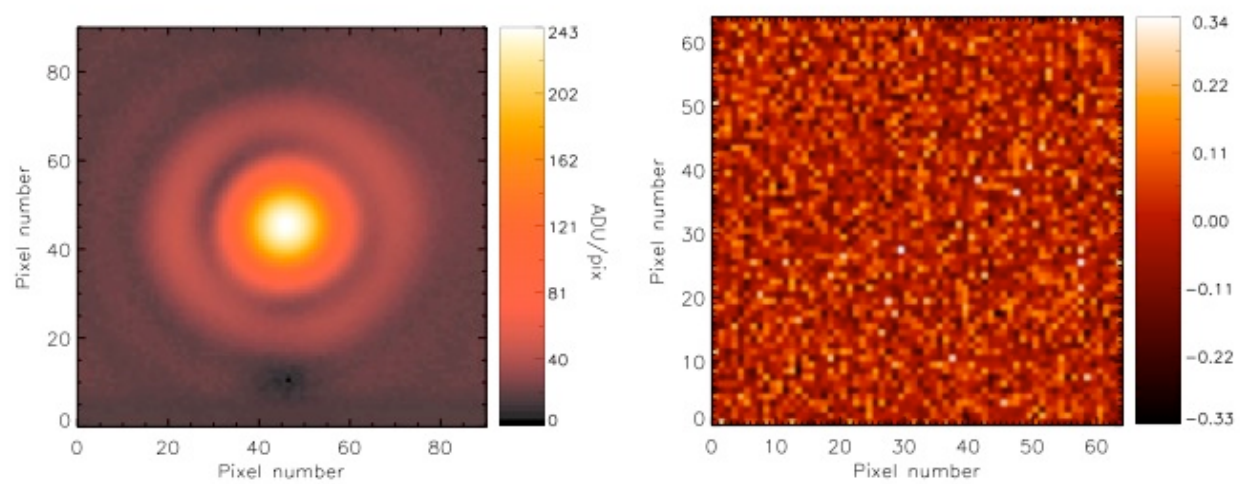

Figure 3.3. Median-combined $11 \mu \mathrm{m}$ image of 15972 frames at $55 \mathrm{msec}$ each with an off-source nod beam subtracted. The star is Vega with a non-linear stretch to show the diffraction rings. The noise is from the same image away from the star with a linear stretch.
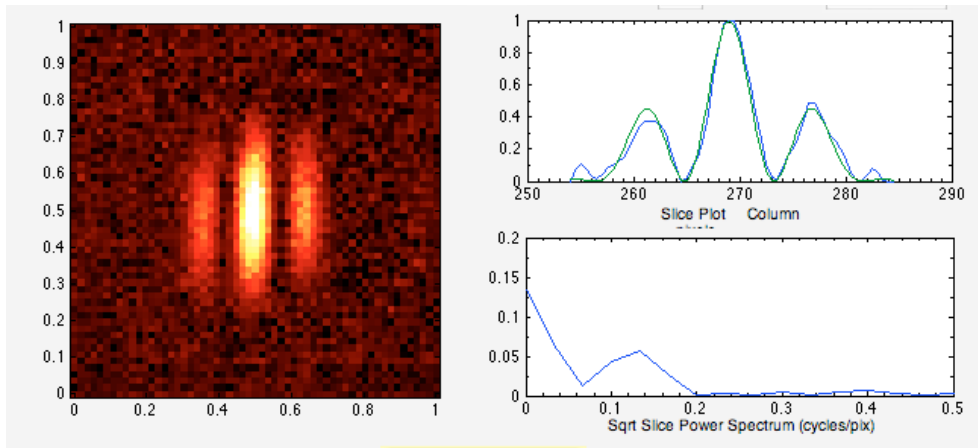

Figure 3.4. Fizeau Interferometry at $11 \mu \mathrm{m}$ wavelength. The left image is a $30 \mathrm{msec}$ exposure of Vega. The upper right plot shows a cut through the image (blue line) that is consistent with a theoretical PSF (green line). The lower right plot is the power spectrum of the image cut. 


\section{LOW FREQUENCY EXCESS NOISE (ELFN)}

Low Frequency Excess noise is a characteristic of Si:As IBC IR arrays. It has been known for many years and affects the devices of all manufacturers of these arrays. ELFN characteristics are:

- ELFN is not noticeable in a single array read. It requires many coadds to see.

- It appears at low frequencies, $<10 \mathrm{~Hz}$

- It is not $1 / \mathrm{f}$ noise.

- It rises above the shot noise approximately a factor of two to five over about a factor of 100 in frequency

- The rise starts at a "knee" which is at a higher frequency for higher incident photon flux

Figure 4-1 shows the ELFN noise for the Aquarius array as a function of frequency. It shows flat noise at high frequencies, then a slow rise a factor of greater than two from $\sim 2 \mathrm{~Hz}$ to $0.006 \mathrm{~Hz}$. For previous generations of IR telescopes with rapid secondary mirror beam switching ELFN was not a problem. For current and future generations of large telescopes beam switching is generally much slower than $10 \mathrm{~Hz}$ so that observing strategies must be adapted to minimize this effect.

\subsection{Adding Spatial Filtering to Noise Measurement}

The noise for Figure 4-1 was calculated as the standard deviation of all the pixels over the array. This is not an appropriate measurement of noise when the energy from a star falls on a number of pixels. The values for these pixels must be added to detect and determine the flux from a star. In addition, in order to remove the effect of possible variation of the background over the array, a region outside the star is frequently subtracted, such as a neighboring area or an annulus.

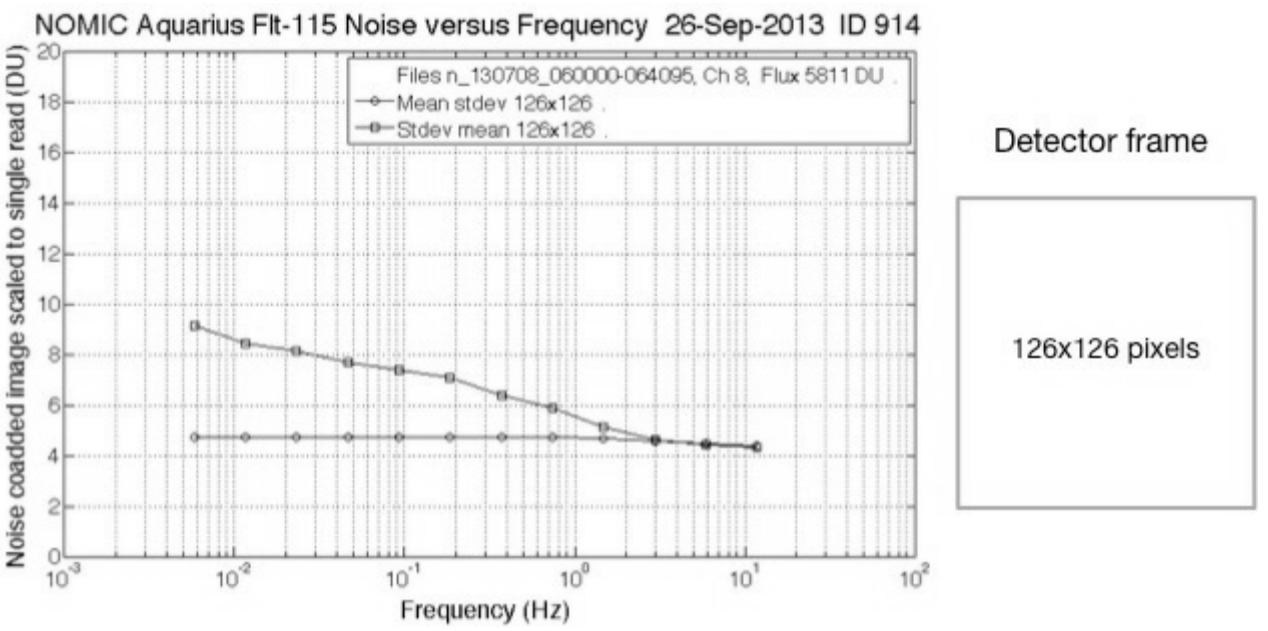

Figure 4-1 Plot of the standard deviation over the array of 2048 126x126 pixel image difference pairs as a function of the frequency calculated from the time interval between pairs. The lower curve is the mean of the standard deviation for single pairs. The upper curve is the standard deviation for 2048 co-added pairs. 


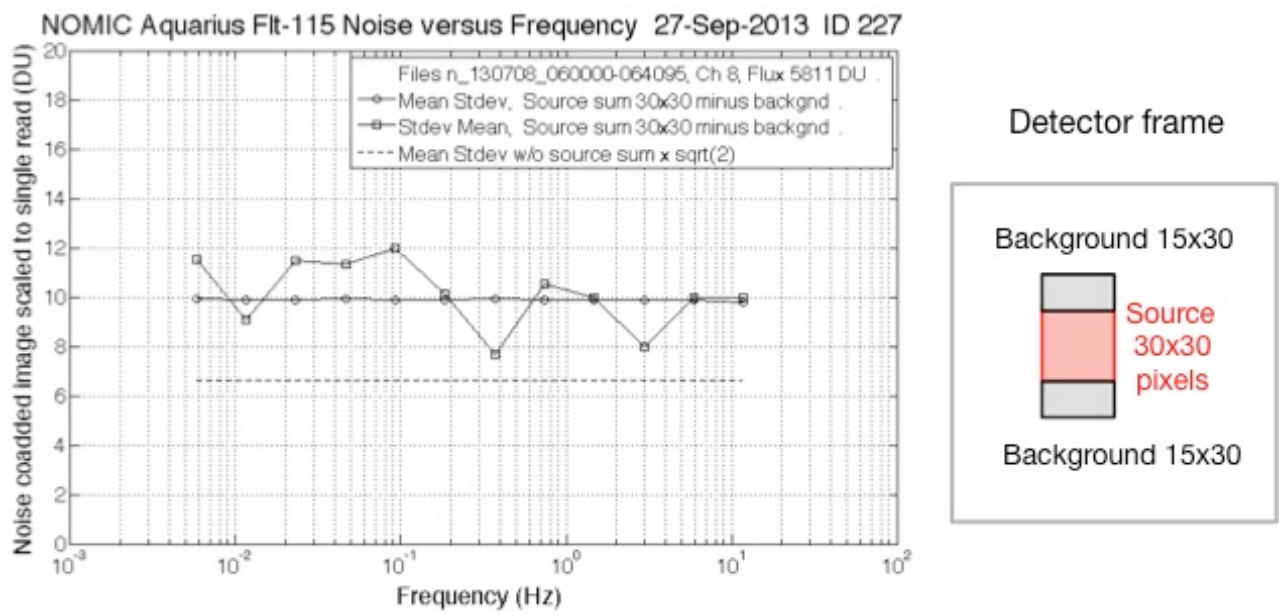

Figure 4-2 Plot of the standard deviation of $2 \times 4$ "pixel" difference pairs for source sum and background subtract as a function of the frequency calculated from the time interval between pairs. The flat curve is for single pairs. The irregular curve is for 2048 co-added pairs. The dashed line is the level expected if the noise of the mean over the photometric aperture decrease and the sqrt(number of pixels),

Figure 4-2 is a plot of the noise versus frequency with the noise calculated as the standard deviation of a set of photometric measurements. Each is the mean of the values of the pixels in a square of a size which would encompass the core of a star minus the mean of background rectangles above and below the square with the same total area. The noise has been normalized by the square root of the number of pixels in the photometric square to compare directly with the noise in Figure 4-1. The scatter is large because there is space in the image for only a few of these photometric areas. The noise is somewhat higher than it would be if the pixel noise over the aperture were completely random and not spatially correlated.

\subsection{Results of Spatial Filtering and Future Tasks}

The effect of this spatial filtering is to largely eliminate the ELFN rise toward the low frequencies suggesting that the ELFN is a high-spatial frequency noise. However, the magnitude of the noise is still greater than it would be without ELFN. The dashed line in Figure 4-2 is the single image noise in Figure 4-1 times sqrt(2) to allow for the subtraction of the background area from the photometric square. The noise in Figure 4-2 is approximately 1.5 times this, a likely consequence of the high spatial frequency correlation of the pixels in the image. The task remains to understand and eliminate this correlation, either by change in the array fabrication or by post image processing.

\section{ACKNOWLEDGEMENTS}

We thank Gert Finger and Derek Ives of the ESO Instrumentation Division for their leadership in the creation and testing of the Aquarius array and their help introducing us to this array. We thank Raytheon Vision Systems for designing and fabricating the array ${ }^{8}$. We thank the Sofia Forcast instrument group at Cornell University for their help.

The LBT Interferometer and camera are supported by NASA through JPL subcontract 1226582.

\section{REFERENCES}

[1] Esposito, S., Riccardi, A., Pinna, E., Puglisi, A., Quiro's-Pacheco, F., Arcidiacono, C., Xompero, M., Briguglio, R., Agapito, G., Busoni, L., Fini, L., Argomedo, J., Gherardi, A., Brusa, G., Miller, D., Guerra, J. C., Stefanini, P., and Salinari, P., "Large Binocular Telescope Adaptive Optics System: new achievements and perspectives in adaptive optics," Proc. SPIE 8149 (2011). 
[2] Hinz, P.M., Connors, T., McMahon, T. Cheng, A., Peng, C.Y., Hoffmann, W., McCarthy, D.J., Angel, R., "Large Binocular Telescope Interferometer: the universal beam combiner" Proc. SPIE 5491, 787 (2004).

[3] Hinz, P., Arbo, P., Bailey, V., Connors, T., Durney, O., Esposito, Simone, Hoffmann, W., Jones, T., Leisenring, J., Montoya, M. et al., "First AO-corrected interferometry with LBTI: steps towards routine coherent imaging observations" Proc. SPIE 8445, 84450U-84450U-10 (2012).

[4] LBTI web site: lbti.as.arizona.edu

[5] Hinz, P. M., Solheid, E., Durney, O., and Hoffmann, W. F., "NIC: LBTI's nulling and imaging camera," Proc. SPIE 7013 (2008)

[6] Wilson, J. C., Hinz, P. M., Skrutskie, M. F., Jones, T., Solheid, E., Leisenring, J., Garnavich, P., Kenworthy, M., Nelson, M. J., and Woodward, C. E., "LMIRcam: an L/M-band imager for the LBT combined focus," Proc. SPIE 7013 (2008).

[7] Pirger, B. E., Schoenwald, J., Herter, T. L., Gull, G. E., Adams, J. D., Keller, L. D., Berthoud, M., Henderson, C., Stacy, G. J., Nikola, T., "High-speed highly flexible reconfigurable data acquisition system for astronomy," Proc. SPIE 6276 (2006)

[8] Mills, R., Beuville, E., Corrales, E., Hoffman, A., Finger, G., Ives, D., "Evolution of Large Format Impurity Band Conductor Focal Plane Arrays for Astronomy Applications" Proc. SPIE 8154 (2012) 